\title{
Micro-Photoluminescence Studies of CdSe/ZnSe Quantum Dot Structures Grown under Different Conditions
}

\author{
M.V. Rakhlin*, S.V. Sorokin, I.V. Sedova, A.A. Usikova, S.V. Gronin, K.G. Belyaev, \\ S.V. IVAnov, A.A. TOROPOV \\ Ioffe Institute, Polytekhnicheskaya 26, St. Petersburg 194021, Russia
}

\begin{abstract}
We report on comparative studies of CdSe/ZnSe quantum dot structures grown by molecular beam epitaxy either with or without predeposition of a sub-monolayer-thick CdTe layer (stressor). Also we consider the structure grown in a thermal activation mode. Emission properties of individual quantum dots are investigated by microphotoluminescence spectroscopy using $500 \mathrm{~nm}$ apertures opened in a non-transparent gold mask. The density of emitting quantum dots and the spectral width of the single-dot emission lines are estimated.
\end{abstract}

DOI: 10.12693/APhysPolA.129.A-117

PACS: 68.65.Hb, 78.55.Et, 81.07.Ta

\section{Introduction}

In the last decade, the physics of quantum light sources, namely, the sources of single photons and entangled pairs of single photons, has become one of the leading areas of nanophotonics and nanoelectronics. Up to now, single photon emission at room temperature has been achieved in molecules [1] and color centers in diamond [2], where serious difficulties exist in fabrication of the electrically driven devices. Single self-organized quantum dots (QDs) grown by epitaxial techniques are good candidates for the electrically driven single photon sources, because they can be easily integrated into commercial diode structures. However, there are very few reports on single photon emission in the epitaxial QDs at room temperature, which is mainly hampered by the relatively low quantum efficiency and spectral overlap of the emission bands of different QDs due to their large density. Bayer and Forchel [3] were able to detect single photon emission in InGaAs QDs at room temperature, however, the integration time in their experiments amounted several hours. The structures based on II-VI wide-bandgap compounds are a good alternative. In a $\mathrm{Zn}(\mathrm{Cd}, \mathrm{S})$ Se system, for instance, strong carrier confinement and reasonable quantum efficiency could be observed up to room temperature [4-6].

Another promising application of QDs is development of efficient laser heterostructures based on either III$\mathrm{V}$ [7] or II-VI compounds [8]. In contrast to the QD single-photon sources, such applications require a possibly large QD density and homogeneity of their sizes and composition.

In this paper, we perform comparative studies of three different types of $\mathrm{CdSe} / \mathrm{ZnSe}$ QDs grown by molecular beam epitaxy (MBE). In particular, we define the types of QD structures, which are preferable either for

*corresponding author; e-mail: maximrakhlin@mail.ru achieving the single photon emission or development of the active region of efficient lasers.

\section{Experiment}

We present experimental data on three characteristic samples (labeled further A, B, and C) grown by MBE on GaAs (100) substrates. The substrate is overgrown by a $0.2 \mu \mathrm{m}$ thick GaAs buffer and then, through a coherent heterovalent interface [9], by either $70 \mathrm{~nm}$ thick (samples $\mathrm{B}$ and $\mathrm{C}$ ) or $50 \mathrm{~nm}$ thick (sample A) ZnSe barriers. For all samples, CdSe QDs were grown using a migrationenhanced epitaxy (MEE) technique [10] for the purpose of improving uniformity of size and composition within the QD ensemble. Sample B was grown with predeposition of a sub-monolayer-thick CdTe layer (stressor) [11], and QDs in sample $\mathrm{C}$ were grown in a thermal activation mode. The latter method implies a low temperature $\left(T_{\mathrm{S}}=230^{\circ} \mathrm{C}\right)$ layer-by-layer growth of CdSe on the $\mathrm{ZnSe}$ surface, followed by a gradual increase in $T_{\mathrm{S}}$ up to $310^{\circ} \mathrm{C}$, carried out under Se flux. This technique is aimed at changing the surface energy and inducing the 2D-3D transition of the strained CdSe layer [12]. The CdSe QDs are capped with a ZnSe layer of a thickness $20 \mathrm{~nm}$ (sample A), $7 \mathrm{~nm}$ (sample B), and $25 \mathrm{~nm}$ (sample C).

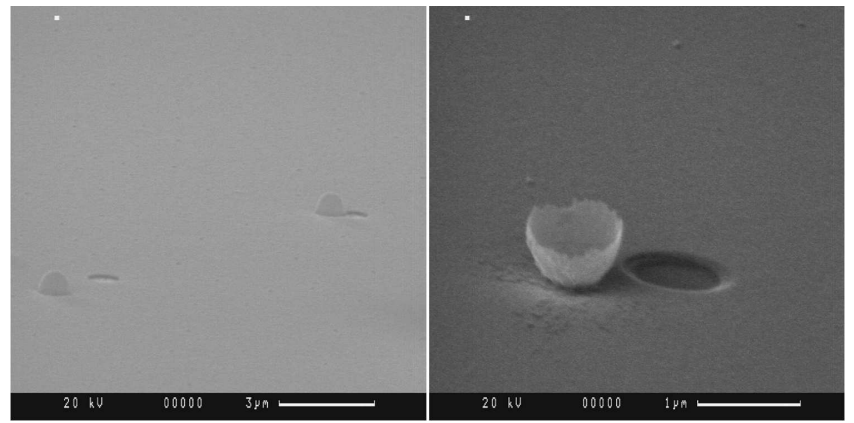

Fig. 1. SEM images of the $500 \mathrm{~nm}$ apertures in nontransparent gold mask, opened by a ball-assisted etching technique. 
Emission properties of individual QDs were investigated using $500 \mathrm{~nm}$ apertures in a non-transparent gold mask, opened by a ball-assisted etching technique. To this end, the as-grown samples were exposed to a diluted water solution of latex balls of $500 \mathrm{~nm}$ in diameter. Then an opaque gold film was deposited above the surface with the precipitated balls, and whereupon the balls were dissolved using 3-chloroethylene. The obtained apertures are shown in Fig. 1. The samples were mounted in a Heflow cryostat. Micro-photoluminescence ( $\mu$-PL) measurements were carried out by optical exciting the samples with a continuous-wave (cw) laser (404 nm). The exciting light is focused by a $50 \times$ microscope objective to a spot of $\approx 20 \mu \mathrm{m}$ in diameter. Then the luminescence is collected by the same objective, passed through a pinhole, dispersed by a $0.5 \mathrm{~m}$ monochromator with a $1200 / \mathrm{mm}$ grating, and detected by a cooled charge coupled device.

\section{Results and discussion}

Figure 2 shows $\mu \mathrm{PL}$ spectra of samples $\mathrm{A}, \mathrm{B}$, and $\mathrm{C}$, measured within the $500 \mathrm{~nm}$ apertures at $5 \mathrm{~K}$. Each spectrum displays a number of overlapping narrow PL lines assigned to the emission of individual QDs or certain groups including a few QDs. The total PL spec-

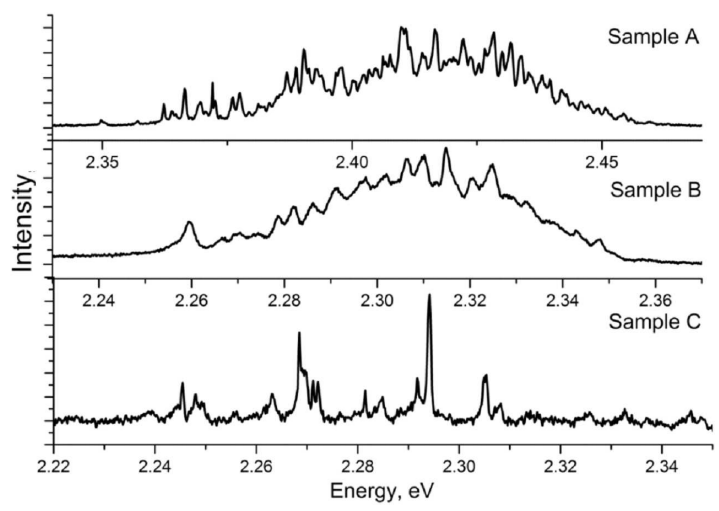

Fig. 2. $\mu \mathrm{PL}$ spectra of samples $\mathrm{A}, \mathrm{B}$, and $\mathrm{C}$ at $5 \mathrm{~K}$ under $c w$ laser excitation $(\lambda=404 \mathrm{~nm})$ measured within the $500 \mathrm{~nm}$ apertures opened in a non-transparent gold mask.

trum of sample B revealed the smaller full width at half maximum (FWHM) than in sample A (35 meV versus $50 \mathrm{meV}$ in sample $\mathrm{A}$ ), that indicates better homogeneity of the QD ensemble in the sample with the stressorcontrolled QD growth, as illustrated in Fig. 3. The wide emission band between 1.7 and $2.2 \mathrm{eV}$ is attributed to a relatively homogeneous thin ZnTeSe layer located between the Cd-enriched islands [11]. At the same time, FWHM of the spectrum of sample $\mathrm{C}$ is about $85 \mathrm{meV}$, which means stronger variation of the emission energies of individual QDs. The $\mu \mathrm{PL}$ spectrum of sample $\mathrm{C}$ shows well-separated narrow PL lines with almost complete absence of background luminescence, that indicates a much lower density of QDs in the structure with in situ annealing as compared with other two samples. We calculated the ballpark numbers of individual PL peaks in the $\mu \mathrm{PL}$ spectra and thus estimated the approximate density of emitting QDs. This density is $7 \times 10^{9} \mathrm{~cm}^{-2}$ in sample A, $1.1 \times 10^{10} \mathrm{~cm}^{-2}$ in sample $\mathrm{B}$, and $1.8 \times 10^{9} \mathrm{~cm}^{-2}$ in sample $\mathrm{C}$. In the samples $\mathrm{A}$ and $\mathrm{B}$, the obtained density is most probably strongly underestimated, especially in sample B, due to emergence of background luminescence and possible emission of biexcitons and trions. The average FWHM of each line in samples A and $\mathrm{C}$ is in the range $0.3-0.6 \mathrm{meV}$. These values fit the data obtained in most of previous studies of single CdSe QDs [5, 13]. In sample B, FWHM of each line has never been less than $1 \mathrm{meV}$, being typically larger than $2-3 \mathrm{meV}$. A possible reason of the enhanced spectral broadening may be the spectral diffusion induced by trapping of the charge carries in the type-II ZnSe/CdTe nanostructures formed presumably in the vicinity of the CdSe QDs [11].

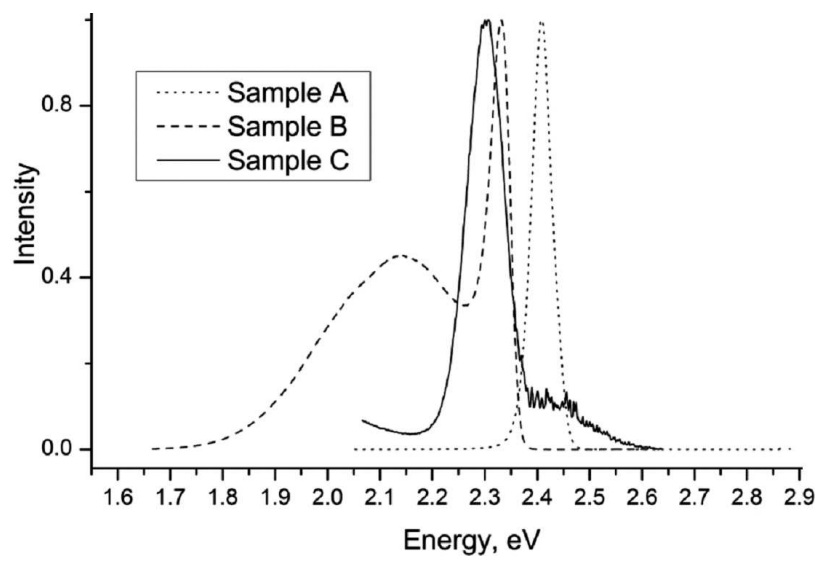

Fig. 3. PL intensities of samples $\mathrm{A}, \mathrm{B}$, and $\mathrm{C}$ at $5 \mathrm{~K}$ normalized to the maximum intensity of the respective sample.

\section{Conclusions}

We have performed comparative studies of $\mu \mathrm{PL}$ spectra in $\mathrm{CdSe} / \mathrm{ZnSe} \mathrm{QD}$ structures grown by MBE under different conditions. The emission spectrum of CdSe/ZnSe QDs with in situ annealing shows a set of narrow peaks $(0.3-0.6 \mathrm{meV})$ without any background luminescence. The density of emitting individual QDs in this sample is estimated as less than $1.8 \times 10^{9} \mathrm{~cm}^{-2}$. In contrast to that, the CdSe QDs grown with the CdTe stressor have demonstrated much larger density of emitting QDs and better homogeneity of the QDs ensemble with FWHM of the total PL spectrum as small as $35 \mathrm{meV}$. These data allowed us to conclude that the CdSe/ZnSe QDs grown in a thermal activation mode suit better for single photon applications, while the QDs grown with the CdTe stressor could be preferable for laser applications.

\section{Acknowledgments}

This work was supported by Russian Science Foundation (Project \#14-22-00107). 


\section{References}

[1] B. Lounis, W. Moerner, Nature 407, 491 (2000).

[2] T. Babinec, B. Hausmann, M. Khan, Y. Zhang, J. Maze, P. Hemmer, M. Loncar, Nat. Nanotechnol. 5, 195 (2010).

[3] M. Bayer, A. Forchel, Phys. Rev. B 65, 041308 (2002).

[4] F. Gindele, K. Hild, W. Langbein, U. Woggon, Phys. Rev. B 60, R2157 (2002)

[5] K. Sebald, P. Michler, T. Passow, D. Hommel, G. Bacher, A. Forchel, Appl. Phys. Lett. 81, 2920 (2002).

[6] O. Fedorych, C. Kruse, A. Ruban, D. Hommel, G. Bacher, T. Kummel, Appl. Phys. Lett. 100 , 061114 (2012).

[7] D. Bimberg, M. Grundmann, N.N. Ledentsov, Quantum Dot Heterostructures, Wiley, Chichester 1999.

[8] S.V. Ivanov, A.A. Toropov, S.V. Sorokin, T.V. Shubina, I.V. Sedova, A.A. Sitnikova, P.S. Kop'ev, Zh.I. Alferov, A. Waag, H.-J. Lugauer, G. Reuscher, M. Keim, G. Landwehr, Appl. Phys. Lett. 74, 498 (1999).
[9] S.V. Gronin, I.V. Sedova, S.V. Sorokin, G.V. Klimko, K.G. Belyaev, A.V. Lebedev, A.A. Sitnikova, A.A. Toropov, S.V. Ivanov, Phys. Status Solidi C 9, 1833 (2012).

[10] I.V. Sedova, S.V. Sorokin, A.A. Sitnikova, O.V. Nekrutkina, A.N. Reznitsky, S.V. Ivanov, Inst. Phys. Conf. Ser., No. 174, Eds. M. Ilegems, G. Weimann, J. Wagner, 2003, Sect. 3, p. 161.

[11] A.A. Toropov, I.V. Sedova, O.G. Lyublinskaya, S.V. Sorokin, A.A. Sitnikova, S.V. Ivanov, J.P. Bergman, B. Monemar, F. Donatini, Le Si Dang, Appl. Phys. Lett. 89, 123110 (2006).

[12] I. Robin, R. Andre, C. Bougerol, T. Aichele, S. Tatarenko, Appl. Phys. Lett. 88, 233103 (2006).

[13] J.C. Kim, H. Rho, L.M. Smith, H.E. Jackson, S. Lee, M. Dobrowolska, J.K. Furdyna, Appl. Phys. Lett. 75, 214 (1999) 